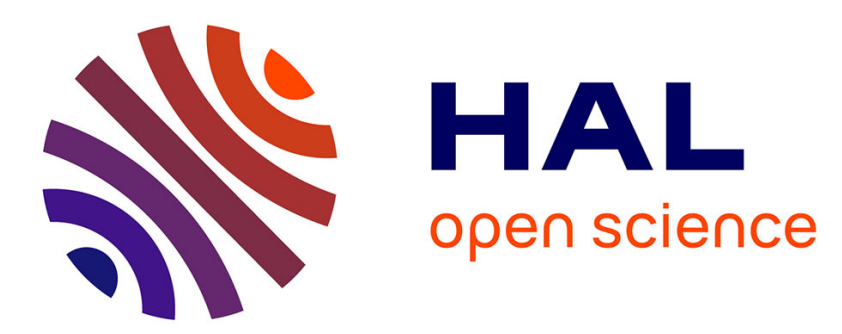

\title{
EXAFS evidence for local order in the ionic aggregates of halato-telechelic polymers
}

\author{
R. Jérome, G. Vlaic, C.E. Williams
}

\section{To cite this version:}

R. Jérome, G. Vlaic, C.E. Williams. EXAFS evidence for local order in the ionic aggregates of halato-telechelic polymers. Journal de Physique Lettres, 1983, 44 (17), pp.717-723. 10.1051/jphyslet:019830044017071700 . jpa-00232255

HAL Id: jpa-00232255

https://hal.science/jpa-00232255

Submitted on 1 Jan 1983

HAL is a multi-disciplinary open access archive for the deposit and dissemination of scientific research documents, whether they are published or not. The documents may come from teaching and research institutions in France or abroad, or from public or private research centers.
L'archive ouverte pluridisciplinaire HAL, est destinée au dépôt et à la diffusion de documents scientifiques de niveau recherche, publiés ou non, émanant des établissements d'enseignement et de recherche français ou étrangers, des laboratoires publics ou privés. 
Classification

Physics Abstracts

$61.40 \mathrm{~K}-61.10 \mathrm{~F}$

\title{
EXAFS evidence for local order in the ionic aggregates of halato-telechelic polymers
}

\author{
R. Jérome $\left({ }^{1}\right)$, G. Vlaic $\left({ }^{2}\right)$ and C. E. Williams $\left({ }^{3}\right)$ \\ ( $\left.{ }^{1}\right)$ Laboratoire de Chimie Macromoléculaire et de Catalyse Organique, \\ Université de Liège, Sart-Tilman, 4000 Liège, Belgium \\ ( ${ }^{2}$ ) L.U.R.E., Orsay et Istituto Guido Donegani, Centro Ricerche, Novara, Italy. \\ $\left(^{3}\right)$ L.U.R.E., Orsay et Physique de la Matière Condensée, Collège de France, \\ Place Marcellin Berthelot, 75005 Paris, France
}

(Reçu le 10 mai 1983, accepté le 6 juillet 1983)

\begin{abstract}
Résumé. - Les agrégats ioniques formés dans du polybutadiène carboxyl téléchélique solide, un polymère ionique aux propriétés moléculaires bien contrôlées, ont une structure locale très ordonnée comme le montrent les mesures d'absorption EXAFS. Pour des échantillons neutralisés avec une proportion de 1 ou $2 \mathrm{Zn}$ par chaîne, chaque métal est entouré en moyenne de 6 atomes d'oxygène mais surtout d'une $2^{\mathrm{e}}$ couche pouvant contenir jusqu'à 5 atomes de $\mathrm{Zn}$, à une distance de $3,22 \pm 0,02 \AA$. L'influence des conditions de préparation et d'élaboration des échantillons est aussi étudiée.
\end{abstract}

\begin{abstract}
The ionic aggregates in bulk halato-telechelic polymers, a class of ion-containing polymers with well controlled molecular characteristics, have a high degree of local order, as shown by EXAFS spectroscopy. In zinc neutralized carboxy telechelic polybutadiene each cation is surrounded by an average of 6 oxygen neighbours as well as by a second shell of up to 5 zinc atoms at a distance of $3.22 \pm 0.02 \AA$. The influence of humidity during sample preparation and elaboration is also investigated.
\end{abstract}

\section{Introduction.}

Ion aggregation is recognized as being the reason for the unique mechanical and transport properties of ion containing polymers [1]. However the detailed structure of the ionic aggregates as well as their organization in the bulk material is still in question. To elucidate the first problem, local methods of investigation have been used like ${ }^{23} \mathrm{Na}$ nuclear magnetic resonance [2], infra-red absorption measurements [3], Mössbauer spectroscopy [4, 5]. Very recently EXAFS (extended $\mathrm{X}$-ray absorption fine structure) experiments $[6,7]$ have shown a well-ordered local structure around the cation in sulfonated polystyrene ionomers and in Nafion ${ }^{\mathbf{R}}$ ionomers. These ionomers consist of a non-polar polymeric backbone with pendant ionic groups, of various complexity, randomly distributed along the chain and their molecular properties are usually not well defined. 
One way to approach the problem is to study compounds whose molecular parameters are well defined and easily varied. Halato-telechelic polymers [8-12] (HTP) form such a class of ion-containing polymers with well controlled molecular characteristics which make them ideal as model compounds for the more complex ionomers. We report here a preliminary study by EXAFS of the local cation environment in carboxy-telechelic polybutadiene neutralized with zinc.

\section{Experimental.}

2.1 Materials. - The $\alpha$, $\omega$-dicarboxylic acid polybutadiene, commercially available from B. F. Goodrich (Hycar CTB 2000X 156; $\bar{M}_{n}=4600$ ) has been neutralized with diethyl-zinc in toluene. $\mathrm{Zn}\left(\mathrm{C}_{2} \mathrm{H}_{5}\right)_{2}$ reacts strongly with carboxylic acid to form the corresponding zinc carboxylate with evolvement of ethane. No secondary reaction product is formed in the reaction medium and neutralization of the carboxylic acid groups is complete.

Three samples have been prepared in different conditions. Samples 1 and 2 have been neutralized under strictly anhydrous conditions according to the following procedure : the carboxytelechelic polybutadiene is initially dried by three successive azeotropic distillations of benzene and finally dissolved in toluene $(5 \% \mathrm{~W} / \mathrm{V})$ previously dried by refluxing over calcium hydride. The appropriate amount of $\mathrm{Zn}\left(\mathrm{C}_{2} \mathrm{H}_{5}\right)_{2}$ in solution in dry toluene is then added dropwise into the carboxy-telechelic polybutadiene, the whole procedure being carried out in nitrogen-purged flasks equipped with rubber septums. When the almost instantaneous reaction is completed, the toluene is removed and the polymer is dried under vacuum until there is no weight reduction. Sample 1 has been neutralized with a stoichiometric amount of $\mathrm{Zn}\left(\mathrm{C}_{2} \mathrm{H}_{5}\right)_{2}$ (i.e. one $\mathrm{Zn}$ atom per difunctional chain or $1.4 \mathrm{Wt} \%$ ) whereas a twofold molar amount of $\mathrm{Zn}(2.8 \mathrm{Wt} \%)$ has been used for sample 2. Sample 3 was also neutralized with a twofold molar excess but in atmospheric conditions, using toluene that had not been previously dried. Of course water in the reaction medium can also react with $\mathrm{Zn}\left(\mathrm{C}_{2} \mathrm{H}_{5}\right)_{2}$ and compete with the $\mathrm{COOH}$ groups. However, since this procedure is simpler it seemed worthwhile knowing if it induces changes in the ion aggregation process. Once in bulk, all samples have been kept under atmospheric conditions but protected from light.

2.2 X-RAY ABSORPTION MEASUREMENTS. - All samples were studied in air at room temperature. The HTP have been molded into $1 \mathrm{~mm}$ thick platelets at $95{ }^{\circ} \mathrm{C}$ for $5 \mathrm{~min}$. and used as such without further annealing. A known amount of finely ground zinc oxide, used as a reference compound of known crystallographic structure (zincite produced by C. Erba ; purity $>99.5$ ) was supported by a $10 \mu$ thick polycarbonate membrane (nucleopore) to form an homogeneous film. The thickness of all samples was adjusted to get the best signal-to-noise ratio.

The EXAFS spectra were recorded at LURE, the Synchrotron Radiation Facility at Orsay, France, on the DCI-EXAFS I spectrometer [13] using a Si(220) channel cut monochromator.

The data were recorded at $2 \mathrm{eV}$ intervals over an energy range of $1,000 \mathrm{eV}$, extending up to $750 \mathrm{eV}$ above the $K$-threshold of zinc $\left(E_{0}=9,659 \mathrm{eV}\right)$; they are described as a measurement of the X-ray linear absorption coefficient $\mu$ in terms of the incident beam intensity $I_{0}$, the transmitted intensity $I$ and the sample thickness $x$, as

$$
\mu x=\ln \left(\frac{I_{0}}{I}\right) .
$$

The EXAFS modulation of the absorption above an absorption edge $E_{0}$ is given by :

$$
\chi(k)=\frac{\mu(k)-\mu_{\mathrm{B}}(k)-\mu_{0}(k)}{\mu_{0}(k)}
$$


where $\mu_{0}$ is the smooth, atomic-like contribution above the edge and $\mu_{\mathrm{B}}$ the background originating from pre-edge absorption processes. $k$ is the photoelectron wave-vector

$$
\left(k=\left[\frac{2 m}{\hbar^{2}}\left(E-E_{0}\right)^{1 / 2}\right]\right),
$$

$E$ is the incident photon energy, $E_{0}$ the photoelectron binding energy, $m$ the electron rest mass and $\hbar$, Planck's constant). For a $K$-edge, the simple back-scattering theory gives the relationship between the EXAFS signal $\chi(k)$ and the structural parameters [9] :

$$
k \chi(k)=\sum_{i} \frac{N_{i}}{R_{i}^{2}} \exp \left(-2 \sigma_{i}^{2} k^{2}\right) F_{i}(k) A\left(R_{i}, k\right) \sin \left[2 k R_{i}+\phi_{i}(k)\right]
$$

where $R_{i}$ is the average distance which separates the absorbing atom from the $N_{i}$ neighbouring atoms defining the $i$ th shell, with a r.m.s. deviation $\sigma_{i} ; \exp \left(-2 \sigma_{i}^{2} k^{2}\right)$ is an effective DebyeWaller factor resulting from the statistical and thermal distortion of the equilibrium structure. $F_{i}(k)$ and $\phi_{i}(k)$ are the amplitude back-scattering factor and phase shift respectively, characteristic of the selected (absorbing atom)-(back-scattering shell $i$ ) pair. The multiplicative factor $A(k)$ takes into account inelastic processes which introduce some loss of coherence of the photoelectrons.

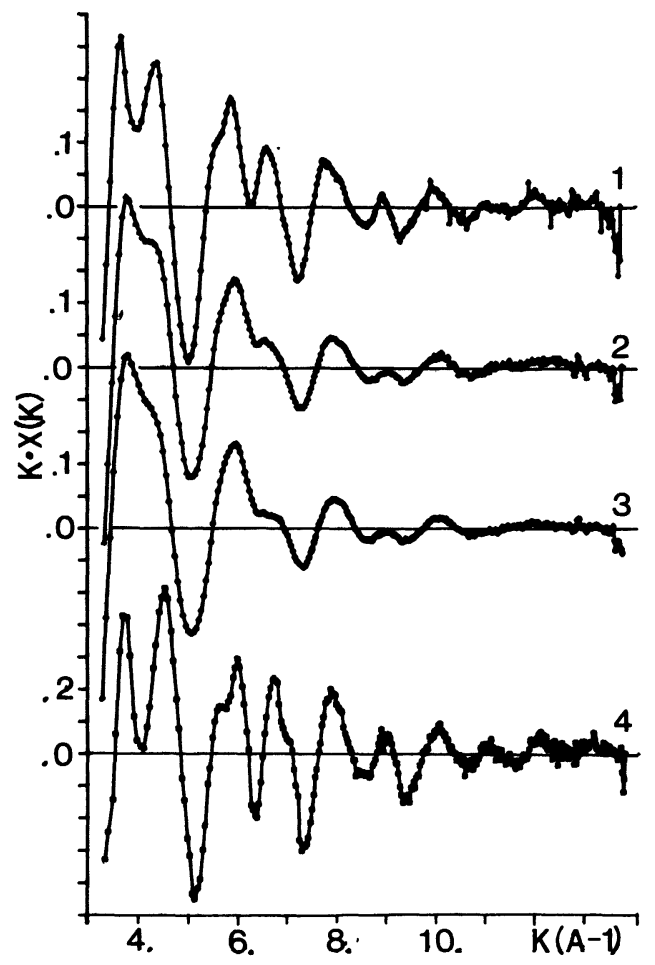

Fig. 1. - The EXAFS oscillations $k \chi(k)$ above the $\mathrm{ZnK}$-edge as a function of the photoelectron wavevector $k$ for the 3 halato-telechelic polymers $(\# 1,2,3)$ and the model compound $\mathrm{ZnO}(4)$. Note the change of scale on the vertical axis for sample 4. 
Data processing was carried out on a UNIVAC 1100/20 computer according to a standard procedure $[14,15]$ that we briefly summarize here :

i) $\mu_{\mathrm{B}}$ is extrapolated from the pre-edge region where a Victoreen fit has been used $\left(\mu_{\mathrm{B}} X=\right.$ $\left.C \lambda^{3}+D \lambda^{4}\right)$ and subtracted from the experimental data. $\mu_{0}(k)$ is first expanded in a fourthorder polynomial in $k$; the slow residual oscillations of $\chi(k)$ are then eliminated by a multiiterative process whose effect appears only in the very small $R$-values (typically $R<1 \AA$ ) of the modulus of the Fourier-transform.

$E_{0}$ is arbitrarily chosen as the inflexion point of the $K$-edge of $\mathrm{Zn}$ in $\mathrm{ZnO}\left(E_{0}=4,965.9 \mathrm{eV}\right)$. The reduced data so-obtained in the terms of formula 2 are shown in figure 1 as a plot of $k \chi(k)$ vs. $\dot{k}$.

ii) The EXAFS spectra are then Fourier-transformed from $k$-space to $R$-space using a Gaussian window which has the value 0.1 at the limits $k_{\min }$ and $k_{\max }$ chosen so as to minimize the loss in resolution. For the Fourier transformation $\chi(k)$ is multiplied by $k^{3}$ so as to give more weight to the high energy part of the EXAFS spectrum whose features could be blurred by the rapid damping of $\chi[16]$. The contribution of each shell of atoms surrounding the absording atom can be isolated in $R$-space. Figure 2 shows the amplitude of the Fourier-transformed spectra $|F T| v s$. $R$, using the window [3.25-13.75] $\AA^{-1}$ for all samples.

iii) The structural parameters (i.e. $R_{i}, N_{i}$ and $\sigma_{i}$ ) can be obtained by least-square fitting the Fourier-filtered spectra of each shell to equation 3 if $F_{i}(k)$ and $\phi_{i}(k)$ are known. For that purpose a physically reasonable model is assumed for each shell and a reference compound of known crystallographic structure is analysed $(\mathrm{ZnO}) . F_{i}(k)$ and $\phi_{i}(k)$ are obtained in a parameterized form $[17,18]$ and transferred to the filtered $|\mathrm{FT}|^{-1}$, allowing a variation of $E_{0}\left(\Delta E_{0}\right)$ to take into account any approximation in this transferrability (the adjusted wave-vector is $k^{\prime}=\left(k^{2}+\right.$ $\left.0.242467 \Delta E_{0}\right)^{1 / 2}$ ).

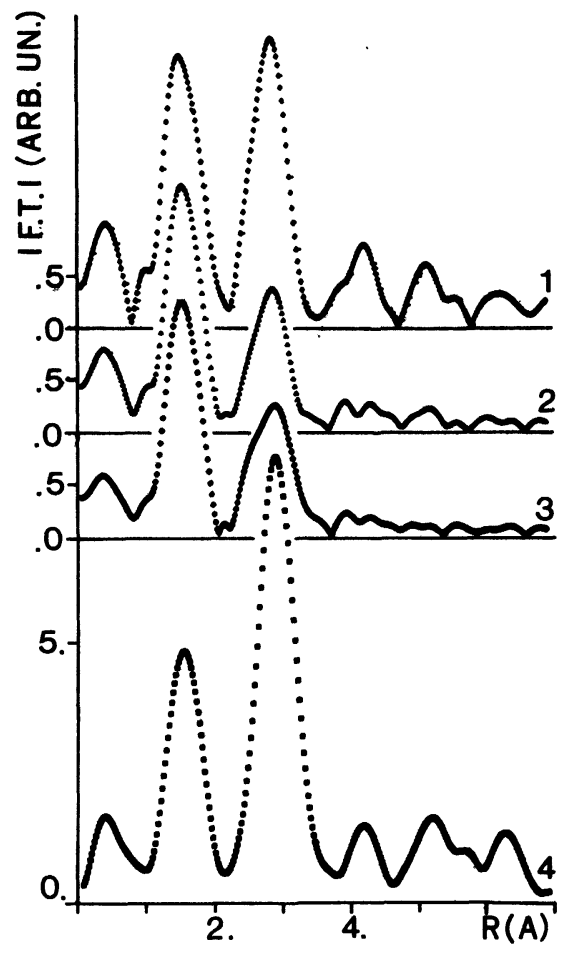

Fig. 2. - Amplitude of the Fourier transforms $\mid$ FT $\mid$ of the EXAFS spectra of figure 1. Same notations as in figure 1. 


\section{Results and discussion.}

Two well-defined shells of atoms surrounding each absorbing $\mathrm{Zn}$ cation are clearly visible for each HTP sample (Fig. 2, curves 1, 2,3); by comparison with $\mathrm{ZnO}$, the first shell has been assigned to $\mathrm{Zn}-\mathrm{O}$ bonds and the second shell to $\mathrm{Zn}-\mathrm{Zn}$ pairs. (As noted earlier, the features below $\sim 1 \AA$ are non physical).

The experimental phases and amplitudes for the $\mathrm{Zn}-\mathrm{O}$ and $\mathrm{Zn}-\mathrm{Zn}$ pairs were obtained by fitting separately in $k$-space the back-transformed contribution $\left(\mathrm{FT}^{-1}\right)$ of the 2 main peaks in the FT of $\mathrm{ZnO}$ (Fig. 2, curve 4), corresponding to 4 oxygen neighbours at an average distance of $1.977 \AA$ and 12 zinc neighbours at a distance of $3.229 \AA$. They were then transferred to the simultaneous $\mathrm{FT}^{-1}$ of the same peaks for the HTP samples and $N, R, \sigma$ and $\Delta E_{0}$ determined by a least-square fitting. The best fits achieved are illustrated in figure 3 and the optimized values are summarized in table I. The precision expected on the bond-length is $\pm 0.01 \AA$ whereas $N$ has an accuracy of the order of $20 \%$.

For all three samples, each $\mathrm{Zn}$ atom is surrounded by a first shell of an average of 6 oxygen atoms at the same average distance as in $\mathrm{ZnO}\left(R_{\mathrm{Zn} . .0}=1.98 \AA\right)$. The room temperature measurements show a larger structural and/or thermal disorder in the first oxygen shell of the polymer than $\mathrm{ZnO}$. Low temperature measurements are planned to assess the importance of statistical structural disorder.

Zinc oxide was chosen as a model compound of known crystallographic structure (wurtzite, $\mathrm{P6}_{3} \mathrm{mc}$ ) to derive empirical phase-shift and amplitude functions for both $\mathrm{Zn}-\mathrm{O}$ and $\mathrm{Zn}-\mathrm{Zn}$ pairs ; these were then transferred to the polymers assuming that they were valid for carboxylate as well as oxide bonds. Let us recall that we cannot derive any information about the crystallographic structure in the aggregates other that it is not of the wurtzite type where $\mathrm{Zn}$ has a 4-coor-

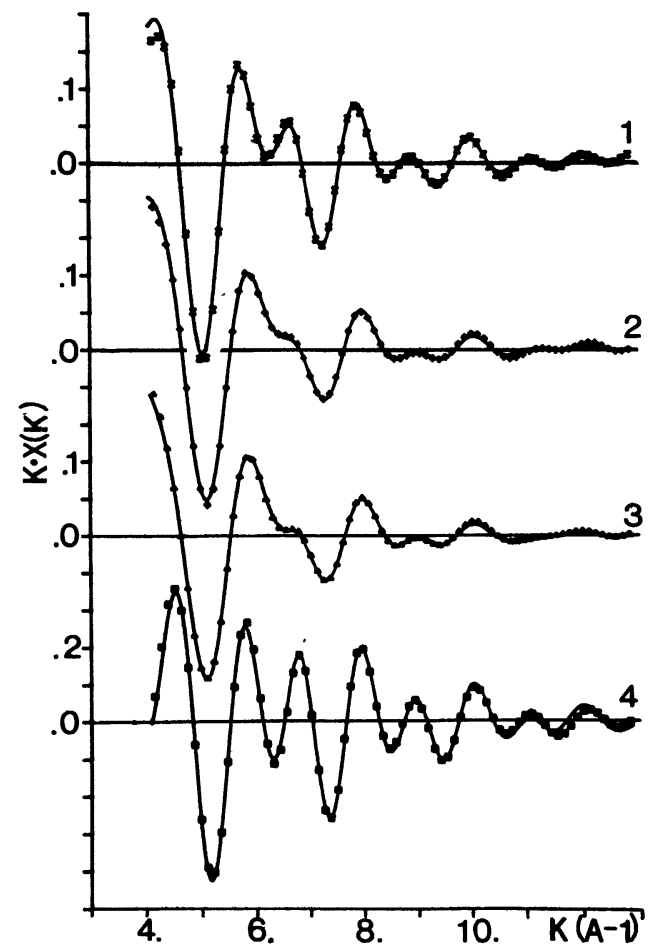

Fig. 3. - Fourier back-transformed data (dots) and best non-linear least square fits for the first two shells contribution for the HTP samples (\# 1, 2, 3) and $\mathrm{ZnO}$ (\# 4). 
Table I. - EXAFS results for the $3 \mathrm{HTP}$ samples in comparison with $\mathrm{ZnO}$.

\begin{tabular}{|c|c|c|c|c|c|}
\hline Sample & Neighbour & $R(\AA)$ & $N$ & $\Delta \sigma(\AA)$ & $\begin{array}{c}\Delta E_{0}(\mathrm{eV}) \\
\left(E_{0}=9664.9 \mathrm{eV}\right)\end{array}$ \\
\hline $\mathrm{ZnO}$ & $\mathrm{O}$ & $\begin{array}{c}1.977 \\
\mathrm{Zn}\end{array}$ & $\begin{array}{c}4.23 \\
12\end{array}$ & - & - \\
\hline $\begin{array}{c}1 \\
1 \text { Zn per chain; } \\
\text { anhydrous }\left(^{*}\right)\end{array}$ & $\mathrm{O}$ & 1.97 & 5.3 & 0.047 & -4.9 \\
\hline $\begin{array}{c}2 \\
2 \mathrm{Zn} \text { per chain; } \\
\text { anhydrous }\end{array}$ & $\mathrm{O}$ & 3.22 & 4.8 & 0.008 & -2.3 \\
\hline $\begin{array}{c}3 \\
2 \text { Zn per chain; } \\
\text { non anhydrous }\end{array}$ & $\mathrm{Zn}$ & 3.20 & 2.6 & 0.013 & -1.0 \\
\hline
\end{tabular}

(*) See text for the sample description.

dination. It could be like the structure of anhydrous or hydrated oxy-salts where $\mathrm{Zn}$ is known to form 6 bonds to oxygen atoms of complex ions or $\mathrm{H}_{2} \mathrm{O}$ molecules.

No significant difference appears in the 1st shell between sample 2, neutralized in anhydrous conditions and sample 3 neutralized without precautions concerning humidity. This might not be surprising since EXAFS does not differenciate between the carboxylate and the oxide bonds and gives an average $\mathrm{Zn}-\mathrm{O}$ bond length. It should be noted however that these two samples give distinctly different Small Angle X-ray Scattering Spectra [20], showing a difference in the organization of the ionic aggregates in the sample, i.e. over a greater distance.

The second shell has been assigned to $\mathrm{Zn}$-Zn pairs with a coordination length similar or slightly shorter than $\mathrm{ZnO}$. This is rather surprising and shows a very compact, well-organized local structure. Let us note that short metal-metal distances have also been measured in nickel Nafions [7] and iron neutralized sulfonated polystyrene [6]. Sample 1, neutralized with a proportion of one $\mathrm{Zn}$ per chain, has an average of five neighbours with no significant difference in disorder, compared with $\mathrm{ZnO}$. It should also be noted (Fig. 2) that sample 1 has a qualitatively similar spectrum at large distances $R>3.5 \AA$ to $\mathrm{ZnO}$ where the signal is dominated by the distant $\mathrm{Zn}-\mathrm{Zn}$ pairs [22]. Although no quantitative result can be derived, it shows that the local order extends beyond the second shell for this sample. This would exclude a model of pseudospherical multiplets of about 8 ion pairs coated by non ionic macromolecular material and grouped into mixed clusters of greater size [1]. A possible way to form large ionic aggregates without chains is to build cylindrical or lamellar domains. We have no evidence yet for such an organization.

Samples 2 and 3, neutralized with an excess of $\mathrm{Zn}$ have a more perturbed structure with an average of 3 like-neighbours.

There is no appreciable difference between samples 2 and 3 except for a more pronounced asymmetry of the second shell (Fig. 2, \# 3) which we have not been able to interpret.

When the $\alpha, \omega$-dicarboxylic acid polybutadiene is neutralized with the stochiometric amount of diethyl zinc (i.e. one $\mathrm{Zn}$ atom per chain, as in sample 1) the metal is expected to be involved 
in the carboxylate groups only (- $\mathrm{COO}-\mathrm{Zn}-\mathrm{OOC}-$ ). It is tied to two chain ends and the structure in the ionic aggregates extends quite far. When a two-fold molar amount of zinc reagent is used, the unreacted alkyl metal bonds $\left(-\mathrm{COO}-\mathrm{Zn}-\mathrm{C}_{2} \mathrm{H}_{5}\right)$ are likely to have been hydrolyzed either during manipulation and molding in air (sample 2) or in the course of synthesis (sample 3) to give $-\mathrm{COO}-\mathrm{Zn}-\mathrm{O}-\mathrm{Zn}-\mathrm{COO}-$. In both cases the $\mathrm{Zn}-\mathrm{Zn}$ pairs should be perturbed, as observed. Still the similar results for the first shell are puzzling.

\section{Conclusion.}

We have shown that the ionic aggregates in solid HTP containing zinc carboxylates are very ordered structures. Each $\mathrm{Zn}$ atom is surrounded by an average of 6 oxygen atoms, at a distance of $1.98 \pm 0.01 \AA$. For the sample neutralized with a proportion of one $\mathrm{Zn}$-atom per chain, each $\mathrm{Zn}$ is surrounded by a second shell of $5 \mathrm{Zn}$ at a well-defined distance of $3.22 \pm 0.02 \AA$ with no significant disorder. The structure is ordered at distances sufficiently large for a 3rd and 4th shell to appear in the EXAFS spectrum, as in $\mathrm{ZnO}$. Neutralization with a higher proportion of $\mathrm{Zn}$ ( $2 \mathrm{Zn}$ per chain) seems to disturb the ordered structure whether water is present or not during the prepolymer neutralization; the second shell is still defined, although with a reduced number of neighbours, at a slightly shorter distance. It should be added that preliminary results on titanium neutralized HTP have also shown the existence of a well-marked second shell of Ti atoms around each $\mathrm{Ti}$.

Future work at low temperature will determine the local order for lower degrees of neutralization.

\section{Acknowledgments.}

The authors wish to thank P. Lagarde for helpful discussions, P. Teyssié for continuing interest throughout this work and the staff of the Laboratoire de l'Accélérateur Linéaire for their assistance with operating the DCI storage ring. Financial support from S.P.P.S. (Brussels) and D.R.E.T. (France) is also gratefully acknowledged.

\section{References}

[1] Eisenberg, A. and King, M., Ion-Containing Polymers (Academic Press, N. Y.) 1977.

[2] Komoroski, R. A. and Mauritz, K. A., JACS 100 (1978) 7487.

[3] Heitner-Wirguin, C., Polymer 20 (1979) 371.

[4] RodmacQ, B. and Pineri, M., Revue Phys. Appl. 15 (1980) 1179.

[5] Meyer, C. T. and Pineri, M., J. Polym. Sci. 16 (1978) 569.

See also RodmacQ, B., Thesis, Grenoble 1982.

[6] Yarusso, D. J., Cooper, S. L., Knapp, G. S., Georgopoulos, P., J. Polym. Sci. 18 (1980) 557.

[7] PAN, H. K., YARUSSO, D. J., KNAPP, G. S. and CoOPER, S. L., to be published.

[8] Broze, G., Jérome, R. and Teyssí,, Ph., Macromolecules 14 (1981) 224.

[9] Broze, G., Jérome, R., Teyssif, Ph. and Marco, C., Polymer Bull. 4 (1981) 241.

[10] Broze, G., Jérome, R., Teyssié, Ph. and Gallot, B., J. Polym. Sci. 19 (1981) 415.

[11] Broze, G., Jérome, R. and TeYssí, Ph., Macromolecules 15 (1982) 920.

[12] Broze, G., Jérome, R. and Teyssí, Ph., Macromolecules 15 (1982) 1300.

[13] Raoux, D. et al., Revue Phys. Appl. 15 (1980) 1079.

[14] Vlaic, G. and Bart, J. C. J., Trav. Chim. 101 (1982) 171.

[15] Vlaic, G. et al., Chem. Phys. 64 (1982) 115.

[16] Stern, E. A., Sayers, D. E. and Lytle, F. W., Phys. Rev. B 11 (1975) 4836.

[17] Lee, B. A., TeO, B. K. and Simmons, A. L., JACS 99 (1977) 3856.

[18] Teo, B. K., Lee, B. A., Simmons, A. L., Eisenberger, P. and KinCade, B. M., JACS 99 (1977) 3854.

[19] SABIne, T. M. and HogG, S., Acta Crystal. B 25 (1969) 2254.

[20] Williams, C. E. and Jérome, R., in preparation.

[21] Goulon, J., Tola, P., Lemonnier, M. and DexPert-Ghys, J., to be published. 